Pharmakologische Mitteilungen.

Volle Beachtung verdient eine Arbeit $\mathrm{Pi}$ a $\mathrm{z} \mathrm{z}$ a s, in der sich der Autor mit den pharmakologischen Wirkungen der Allylverbindungen befaßt ${ }^{38}$ ); haben $j a$ in den letzten Jahren abgesehen von längst bekannten Allylderivaten (Thiosinamin usw.) wieder einige neue Eingang in die Therapie gefunden, wie z. B. das Alival und das Dial. Untersucht wurden Allylaminchlorhydrat, Allylformiat, Allylalkohol, Allylanilin, Allylacetat, Allylessigsäure, Allyljodid, Allylharnstoff, Diallylthioharnstoff, Dithiosinamin, Dimethylallylamin und Diallylbarbitursäure (Dial). Während man bisher auf Grund der Untersuchungen L e v a d i $\mathrm{t}$ is angenommen hat, daß Allylamin ein indifferenter Körper sei, ergaben die Versuche Pi a z.z as, namentlich bei Warmblütern, eine nicht zu vernachlässigende Giftigkeit. Bei Kaninchen genügten Injektionen von $0,1 \longrightarrow 0,2 \mathrm{~g}$ pro Kilogramm Körpergewicht, um Atembeschleunigung, Dyspnöe, Orthopnöe, Stillstand der Atmung und der Herztätigkeit hervorzurufen. Der Tod erfolgte aber nicht durch Atemlähmung, sondern durch Zirkulationsschwäche. Die letale Dosis betrug 0,04 g. Weitere Vergiftungserscheinungen waren Temperatursturz (bis auf $36^{\circ}$ ), Darmreizungen, Hyperämie verschiedener Organe (Lunge, Leber usw.), Hämorrhagien im Ileum und Abnahme des Blutdruckes. Auch das Allylformiat erwies sich als ein pharmakologisch sehr wirksamer Stoff, wenn auch in anderer Richtung wio das Allylamin. So traten bei entsprechenden Dosen die Vergiftungserscheinungen der Leber, Niere und Lunge mehr in den Vordergrund. Ähnlich verbielt sich Allylalkohol, dessen Intoxicationserscheinungen aber einen mehr protrahierten Verlauf nahmen, so daß der Exitus erst nach 3 oder 4 Tagen erfolgte.

Allylanilin erzeugte die typische Anilinvergiftung (Methämoglobinurie), Allylacetat bei Dosen von $0,1 \mathrm{~g}$ pro Kilogramm deutlichen Temperatursturz, während Allylessigsäure bei gleicher Dosierung unwirksam war. Toxischer erwies sich das Allyljodid, das schon bei Dosen von $0,1 \mathrm{~g}$ letal wirkte und außerdem sowohl die Damschleimhaut als auch das Unterhautzellgewebe lokal reizte. Ähnlich wie Allylamin, nur schwächer, wirkte Diallylbarbitursäure bei innerlicher Verabreichung. Bei Kaninchen lag die letale Dosis über 0,12 g pro Kilogramm Körpergewicht. Als unwirksam wurden bei Injektion von $0,1 \mathrm{~g}$ Diallylthioharnstoff, Allylharnstoff, Dithiosinamin und Dimethylallylamin befunden.

Die atembeschleunigende Wirkung des Allylrestes kommt auch in seinen Verbindungen mit atemverlangsamenden Mitteln zum Ausdruck, wie beim N-Allylnorkodein

$$
\mathrm{C}_{16} \mathrm{H}_{14} \mathrm{O} \underset{\mathrm{OH}}{\mathrm{O} \cdot \mathrm{O} \cdot \mathrm{CH}_{3}}
$$

Dieses Präparat bewirkt nach $\mathrm{P}$ o h $\mathrm{1}^{39}$ ) erst nach wiederholten Gaben von $0,04 \mathrm{~g}$ im Tierversuch eine Atembeschleunigung, ja es hebt sogar die Wirkung des Morphins und Heroins auf die Respiration ganz oder teilweise auf, wenn es vor diesen Medikamenten gegeben wird. Bei gleichzeitiger Verabreichung von Morphin und N-Allylnorkodein kommt die Wirkung des Morphins ebenfalls nicht zur Geltung. Es genügen bereits $0,005 \mathrm{~g} \mathrm{~N}$-Allylnorkodein, um den Effekt von $0,03 \mathrm{~g}$ Morphin zu vermindern und die Gefahren des Morphins bezüglich der Atmung zu beseitigen. Ob auch die Wirkung des Morphins auf das Gehirn antagonistisch beeinflußt wird, muß erst noch durch Versuche am Menschen bewiesen werden.

Interessant ist auch eine Arbeit von $\mathrm{H}$ e i m a $\mathrm{n}^{40}$ ), die sich mit der pharmakologischen Wirkung entmethylierter Morphinderivate beschäftigt. Es wird in ihr eine stattliche Anzahl von Norderivaten besprochen, die pharmakologisch beachtenswerte Körper darstellen, wie Normorphin, Norapomorphin, Dihydronormorphin, Norkodein, Benzylnorkodein, Amidonorkodein, Nordionin, Noramylmorphin usw. Die Giftigkeit und Wirksamkeit dieser Norpräparate ist nun gegenüber den entsprechenden Morphinderivaten fast immer vermindert, während $\mathrm{Ehrlich}$ und $\mathrm{Poul}$ s son seiner

38) Z. exper. Path. u. Therap. 17, 318 [1915].

39) Z. exper. Path. u. Therap. 17, 370 [1915].

40) Z. exper. Path. u. Therap. 17, 342 [1915] zeit bei den Cocainen nachgewiesen haben, daß die Entmethylierung Toxizität und Wirksamkeit steigert. Mit Berücksichtigung der oben angegebenen Befunde $\mathbf{P o h} l \mathrm{~s}$ ist es interessant, zu hören, daß beim Normorphin die typische Atemwirkung des Morphins verloren gegangen ist. Sonst konnte bei den Normorphinderivaten im Tierversuch statt einer erregenden eine mehr sedative Wirkung festgestellt werden. Bemerkt sei noch, daß Pentamethylendinormorphin und Dihydronorkodein eine ausgesprochene Darmlähmung verursachen.

[A. 51.]

\section{Über die Veränderungen von Gespinstfasern mit Alkalien und Säuren und deren Folgen für die Textilindustrie.}

\section{Von Dr. Eugen Seel und Dr. Albert Sander, Stuttgart.} (Eingeg. 27./4. 1916.)

Im Verlaufe früherer Untersuchungen über die Haltbarkeit feldgrauer Stoffe ${ }^{1}$ ) fanden wir, daß das Mikroskop bei sehr starken Vergrößerungen wertvolle Aufschlüsse über den Zustand der Faser zu geben vermag, und konnten so unter anderem auch an der Wollfaser die Einwirkung von Ätzalkalien genauer studieren.

Vor kurzem erschien in dieser Zeitschrift ein Bericht über eine neue Untersuchungsmethode zum Nachweis geschädigter Wolle von dem leider inzwischen gefallenen Dr.-Ing. v. A $11 \mathrm{w}$ ö $\mathrm{rd} \mathrm{e} \mathrm{n}^{2}$ ), der ebenfalls auf die große Bedeutung des Mikroskops für die Untersuchung der Wolle hinweist. Auf die sehr interessanten Ausführungen, die zum

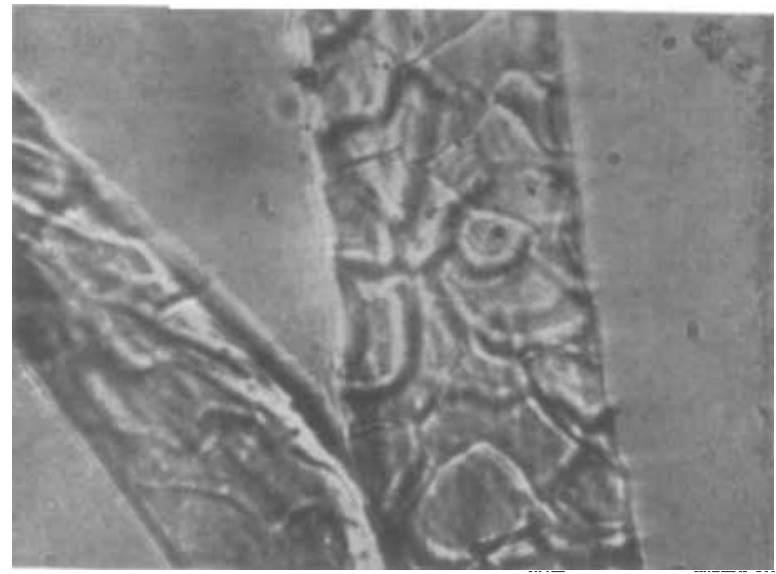

Blld 1. Wollfaser, nicht behandelt. (VergröBerung sãmtlicher Bilder ea. 700 fach.)

Teil frühere Untersuchungen von uns bestätigen, werden wir weiter unten zurückkommen.

Sonst findet man in der einschlägigen Literatur bis jetzt unerwartet wenig Hinweise auf mikroskopische Untersuchungen von Gespinststoffen.

Wir hielten es daher für notwendig, die Veränderungen der Wollfaser durch die bei der Färberei gebräuchlichen Chemikalien eingehender zu verfolgen und die Ergebnisse der Untersuchungen möglichst durch Mikrophotographien festzuhalten.

Nach unseren Erfahrungen bei Wolle lag die Annahme nahe, daß auch bei Gespinstfasern anderer Herkunft, die etwaigen Veränderungen, welche dieselben bei der Einwirkung von Säuren und Alkalien erleiden, ebenfalls noch nicht bei sehr starken Vergrößerungen festgehalten sein könnten.

Vom rein chemischen Standpunkt aus ist dagegen das Verhalten der Faserstoffe gegen Chemikalien bereits eingehender studiert. Die Ergebnisse dieser Untersuchungen sind in zahlreichen Veröffentlichungen niedergelegt.

1) S e e l, Utber die Bekleidungsstoffe und Ausrüstungsstücke der Truppen. Veröff. d. Mil.-San.-Wesens 1914, Heft 62, 103ff.; Angew. Chem. 28, I, 412 u. 428ff., 457 ff.; 29, I, 92ff., 125ff. [1916].

2) Angew. Chem. 29, I, 77-78 [1916]. 
Ausführliche Literaturhinweise findet man in den technologischen Werken und neueren Lehrbüchern. Es sei hier nur auf das 1910 erschienene Werk von $W$ it t und $L$ e h mann: "Chemische Technologie der Gespinstfasern", sowie auf das neuere Buch gleichen Titels von Stirm (Berlin 1913) und das ausführliche Lehrbuch der "Chemie der Cellulose" von C. G. S c h w a l be (Berlin 19I1) hingewiesen.

Auch über die Veränderungen der Faserstoffe durch Chemikalien, insbesondere durch Säuren und Alkalien, in physikalischer (mechanischer) Hinsicht liegen verschiedene Veröffentlichungen vor $^{3}$ ).

Unsere Untersuchungen erstreckten sich auf die drei für das Militärbekleidungswesen wichtigsten Faserstoffe Wolle, Baumwolle und Leinen. Im folgenden sei über die Ergebnisse dieser Untersuchungen berichtet.

\section{Wolle.}

a) Verhalten ge gen Säuren.

Die Einwirkung von Säuren auf die Wollfaser, die einen amphoteren, eiweißartigen Körper darstellt, ist bekannt. Nach Friesen hagen und A p ple yar d $d^{4}$ läßt sich die bei der Behandlung mit verdünnten Säuren von der Wollfaser aufgenommene Säure durch Auswaschen nicht vollständig entfernen.

Gel $\mathrm{mo}$ und $\mathrm{Su}$ i $\mathrm{a}^{5}$ ) fanden, daB die Wolle durch Behandeln mit Säuren in der Wärme an Anfärbbarkeit für basische Farbstoffe einbüßt, daqegen sich mit sauren Farbstoffen tiefer anfärbt.

Die Wirkung verdünnter Säuren soll selbst bei Kochtemperatur durchaus nicht schädigend für die Wollfaser sein. Bei mäßiger Wärme sollen verdünnte Säuren eine Erhöhung der Zugfestigkeit bis zu $7 \%$, nach W i es ne $\mathbf{r}^{6}$ ) sogar bis zu $20 \%$ bewirken. Steigt der Säuregehalt aber über $7 \%$, so findet eine Schädigung statt. Lang anhaltendes Kochen mit mäßig verdünnter Schwefelsäure oder Salzsäure zersetzt die Wollfaser vollständig, wobei als Abbauprodukte Aumoniak, Schwefelwasserstoff, Leucin, Tyrosin, Asparaginsäure und Glutaminsäure auftreten ${ }^{7}$ ). Konzentrierte Säuren zerstören die. Wollfaser schon nach kurzer Zeit.

Wir haben nun die Wolle bei verschiedenen Temperaturen mit verdünnter Schwefelsäure von solchen Konzentrationen behandelt, wie sie für die Färberei in Frage kommen.

Mikroskopisch läßt sich selbst bei 1000 facher Vergrößerung nach dem Kochen mit $1 \%$ iger Säure kein Unterschied gegenüber nicht behandelter Wolle feststellen. Auch zahlreiche Proben aus sauer gefärbten Tuchen sowie aus Chromierfärbungen ließen Strukturveränderungen der Wollfaser selbst bei den stärksten Vergrößerungen nicht erkennen.

Die bei 700 facher Vergrößerung hergestellte Mikrophotographie einer Wollfaser, die 11/4 Stunde mit 1\%iger Schwefelsäure gekocht war, zeigte gegenüber dem bei gleicher Vergrößerung hergestellten Bilde der nicht behandelten Wolle (Bild Nr. I und 10) keine bemerkenswerte Änderung.

b) Verhaltengegen Alkalien.

Viel stärker und schädigender als Säuren wirken Alkalien auf die amphotere Wollfaser ein, namentlich in der Wärme.

Schwächer als die Ätzalkalien wirken ihre Carbonate und die alkalischen Erden. Ammoniak greift Wolle nur in der Wärme und bei sehr starken Konzentrationen merkbar an.

3) Vgl. W. Kind, Ujber den $\operatorname{Einfluß}$ von Wascbmitteln auf Baumwolle und Leinen. Deutsche Färbereizeitung Nr. 4lff. [1908]. - W. K in d, Ưber den Einfluß von Säuren und Alkalien auf die Festigkeit von Baumwolle und Leinen. Ebenda Nr. 29ff [1909]. J. Merrit Matthews, Der Einfluß alkalischer Waschmittel auf die Festigkeit von Wollgarnen. Referat Seifensiederzeitung Heft 30/31. - Einiges über Waschmittel. Referat Seifensioderzeitung 42, 697ff. [1915]; Angew. Chem. 28, II, 628 [1915].

4) S t i r m, Chem. Technol. d. Gespinstfasern S. 151 .

5) Ebenda, S. 152 .

B) W it t, Chem. Technol. d. Gespinstfasern S. 90.

?) Ebenda, S. 90.
Verfolgen wir einmal die fortschreitenden Veränderungen der Faser durch Alkali, wie sie bei 1000 facher Vergrößerung erscheinen:

Die Einwirkung von Alkali auf Wolle läßt sich mikroskopisch besonders anschaulich verfolgen. Wir haben verschieciene Wollsorten cier Einwirkung voll Alkali bei verschiedenen Temperaturen und Konzentrationen bis zur Auflösung unterworfen. Verschiedene Wollsorten, besonders gröbere scheinen etwas widerstandsfähiger gegen Alkali zu sein. Sogenannte Lammwolle wird nach unseren Beob-

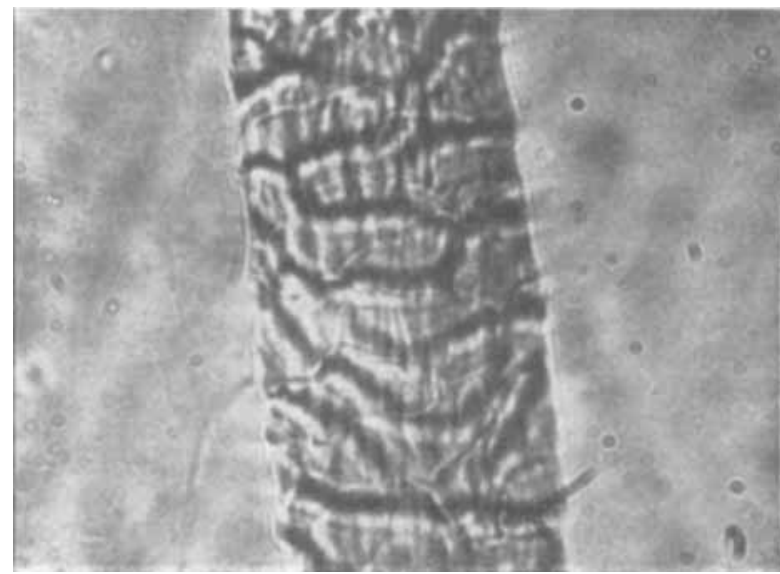

Bild 2. Wollfaser, 1/4 Stunde mit 1\%iger Natronlauge behandelt. Temperatur $40^{\circ}$

achtungen am raschesten und stärksten angegriffen. Das ist wohl auf die größere Feinheit und Lockerheit der betreffenden Faser zurückzuführen.

Die Einwirkung von Alkali macht sich im Mikroskop durch eine Längsfältung der Epithelschuppen bemerkbar, die als Längsstreifung in Bilde erscheint und sich am sinnfälligsten etwa mit den Falten und Runzeln der Schale eines welken Apfels vergleichen läßt.

Das Mikroskop ist bei diesen Beobachtungen scharf auf die Oberfläche des Wollhaares einzustellen. Stellt man mehr auf die Mitte des Faserquerschnittes ein, so könnten die

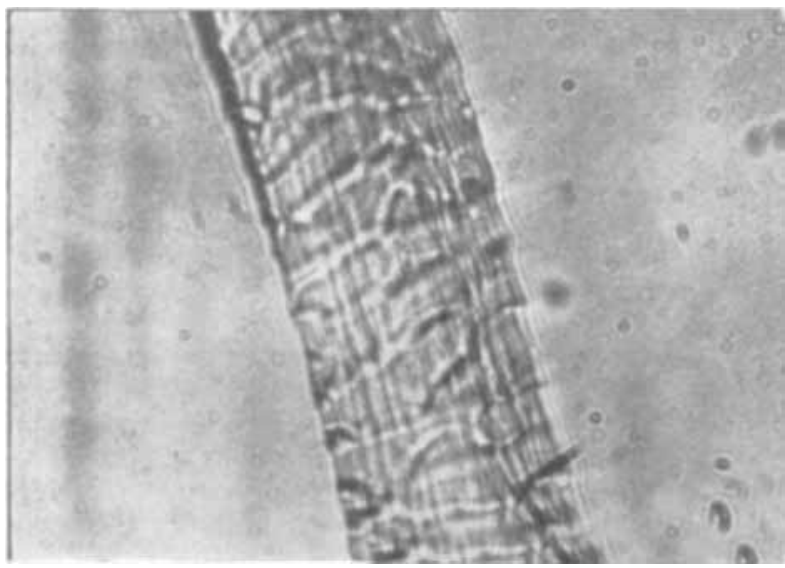

Bild 3. Wollfaser, $1 / \downarrow$ Stunde mit $1 \%$ iger Natronlauge behandelt. Temperatur $50^{\circ}$

durchscheinenden, langgestreckten Zellen der Fas er$\mathrm{s} \mathrm{ch}$ i $\mathrm{ch}$ t ein falsches Bild erwecken und eine in Wirklichkeit nicht vorhandene längsstreifung vortäuschen. (Manche Abbildungen in dèn einschlägigen Handbüchern, z. B. Sti rm, S. 116 und 117 , dürften so entstanden sein und geben daher scheinbar das Bild einer alkalisch behandelten Faser wieder.) Man bettet die Faserobjekte am zweckmäBigsten in Wasser oder für die mikrophotographischen Aufnahmen in Glyceringelatine ein. Zedernöl oder Canadabalsam machen das Präparat zu stark durchscheinend und lassen die feinen Kontraste nicht gut hervortreten.

Da die Mikrophotographien immer nur eine begrenzte Ebene der Faseroberfläche wiedergeben können, empfehlen 
wir Interessenten, die fortschreitenden Veränderungen am Objekt selbst unter fleißiger Benutzung der Mikrometerschraube zu verfolgen. Dann erhält. man ein erschöpfencies Bild von den ganzen Fasererscheinungen

Schon nach $1 / 4$ stündiger Behandlung mit $1 / 2 \%$ iger $\mathrm{Na}-$ tronlauge bemerkt man eine Quellung der Epidermisschicht, die ein Faltenziehen der Epithelschuppen zur Folge hat. Die Falten verlaufen in der Längsrichtung der Faser. Vergleicht man dainit eine unbehandelte Wollfaser, so findet man, da $B$ hier die Epithelschicht glatt und straff verläuft und keine

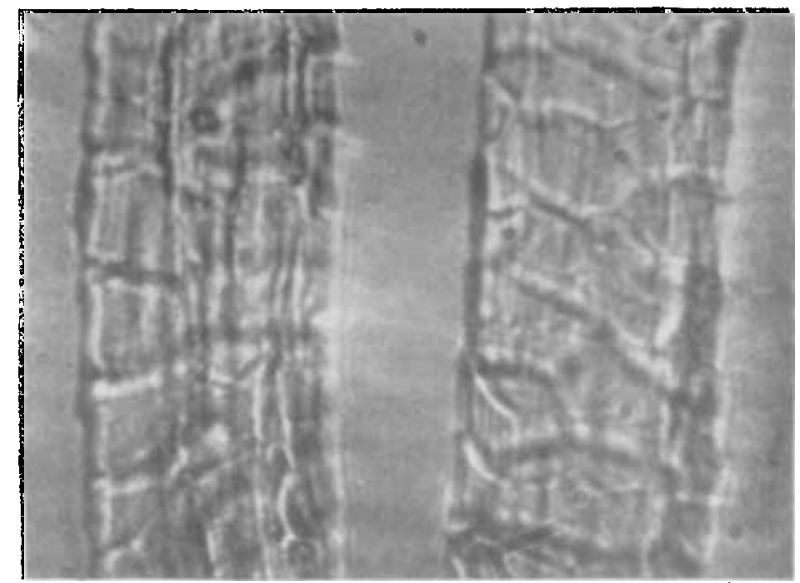

Bild 4. Wolliaser, $1_{4}$ Stunde mit $1 \%$ iger Natronlauge behandelt. Temperatur $60^{\circ}$

Streifung zeigt. Mit steigender Temperatur vertieft sich die Faltung, so da $B$ auch die quer verlaufenden Ränder der Epithelschuppen aus ihrer Richtung verzogen werden. Gleichzeitig wird durch die fortschreitende Auflösung der Epidermis diese dünner und durchscheinenider, so da $\beta$ nun auch die Struktur der Faserschicht immer deutlicher erkennbar wird. Bei weiterem Erwärmen reißt die Epithelschicht an manchen Stellen ein und die inneren Faserzellen quellen hervor. Nun tritt rasch ein Zerfall des Wollhaares in seine Zellelemente ein, und bei $90^{\circ}$ erkennt man im mikro-

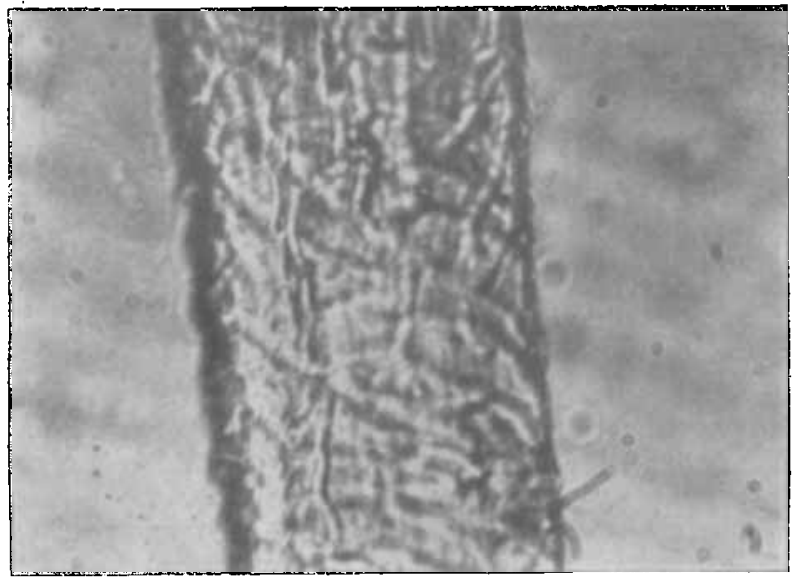

Bild 5. Wollfaser, $1 / 4$ Stunde mit $1 \%$ iger Natronlauge behandelt. Temperatur $70^{\circ}$.

skopischen Bilde nur noch Teile gequollener Faserzellen und Epidermisfetzen.

Wir haben eine Reihe von Mikrophotographien bei 700facher Vergrößerung aufgenommen, welche die fortschreitende Einwirkung von 1\%iger Natronlauge auf die Woll faser recht anschaulich wiedergeben.

Zu diesem Zwecke wurde eine Wollprobe, wie sie für Heerestuche in Anwendung kommt, mit 1\%iger Natronlauge bis zur Auflösung behandelt. Die Behandlung begann bei einer Temperatur von $40^{\circ}$. Nach je 15 Minuten wurde, nachdem man jedesmal eine kleine Faserprobe aus der Lauge heraus genommen und gut abgespült hatte, clie Temperatur des Bades um je $10^{\circ}$ erhöht.
Bild Nr. 1 gibt als Vergleichsobjekt umbehandelte Wollfaser wieder. Man erkennt deutlich die schuppenartigen Epithelzellen. Bild 2 zeigt eine solche Wollfaser nach $1 / 4$ stündiger Behandlung bei $40^{\circ}$. Hier tritt schon deutlich die Falten- und Runzelbildung in der Epithelschicht auf. Die Falten verlaufen in der Längsrichtung der Faser.

Noch deutlicher tritt diese Längsstreifung bei Bild 3 hervor, das nach weiterer $1 /$ stündiger Einwirkung bei $50^{\circ}$ aufgenommen wurde. Die Falten sind im Vergleich zum vorhergehenden Bilde wesentlich vertieft.

Im Bild 4, das die Verhältnisse bei $60^{\circ}$ wiedergibt, erkennen wir bereits tiefe Längsfurchen. Bild 5 , welches einer Temperatur von $70^{\circ}$ entspricht, zeigt den beginnenden Zerfall der Epidermisschicht. Die Ränder der Epithelzellen werden verzogen, und die Schicht selbst wird stark gelockert. Die Faser quillt stark auf.

Den Zustand des Zerfallens der Faser erläutert Bild 6. Hier, bei einer Temperatur von $80^{\circ}$, löst sich die Cuticularschicht von der Faserschicht. Die Faser löst sich in ihre Zellenelemente auf.

Mit Ammoniak unter ähnlichen Bedingungen behandelte Wolle ließ keine derartige Strukturänderungen erkennen.

Wird eine alkalisch behandelte Wolle mit verdünnten Säuren erwärmt, so tritt die Längsstreifung noch deutlicher ein.

Dies zeigen die Bilder 7-9, welche 700 fach vergrößerte Wollfasern wiedergeben, die nach je $1 / 4$ stündiger Behandlung mit $1 \%$ iger Natronlange bei 40,50 und $60^{\circ}$ (wie bei Bild 2 bis 4) $1 \frac{1}{4}$ Stunde mit $1 \%$ iger Schwefelsäure gekocht wurden. Hier ist die Längsstreifung erheblich deutlicher ausgeprägt als vorher. Die bei $60^{\circ}$ alkalisch vorbehandelte Faser beginnt bereits sich aufzulösen. Während also Wolle an sich außerordentlich beständig gegen verdünnte Säuren ist (wie Bild 10 beweist), wird alkalisch vorbehandelte $W$ olle auch von Säuren stark angegriffen. (Die scheinbare Streifung bei diesem Bilde ist deutlich von dem Faltenziehen und der Runzelung der alkalisch behandelten Fasern unterschieden. Vgl. die oben angeführte Bemerkung über die Abbildungen der Handbücher über Textilfasern.)

Bei sorgfältig ausgeführten Küpenfärbungen in ätzalkalischen Küpen, wie sie nach Entdeckung des Hydrosulfits zahlreich in Aufnahme kamen, sind diese Schädigungen wohl praktis ch nicht ins Gewicht fallend, wie ja auch die anerkannte Brauchbarkeit der indigoblauen Uniformen bewiesen hat. Hier ist die Alkalikonzentration ja auch viel geringer und ein Teil des Ätznatrons überdies durch das mildere kohlensaure Alkali oder durch Ammoniak ersetzt. So konnten wir denn auch an solchen Färbungen inder Regel keine Schädigungen in diesem' Sinne feststellen.

Ammoniak greift, wie oben erwähnt, erst bei hoher Konzentration und in der Wärme die Wollfaser merkbar an. So kann man beim Erhitzen von Wolle mit 10\%igem Ammo. niak eine Einwirkung auf die Epithelschicht deutlich erkennen; aber auch schon Wasser soll nach $W$ it $t^{8}$ ) formverändernd auf die Wolle einwirken. Die Epithelschicht soll hier bei längerem Erhitzen ebenfalls faserstreifig und matt und die Zugfestigkeit des Wollhaares geringer werden.

Bei mittleren Temperaturen und mäßiger Konzentration ist durch Ammoniak jedoch keine Schädigung der Wollfaser zu befürchten. (Vergleiche auch die ausführliche $\mathrm{Ab}$ handlung von Dr. B e i $1^{9}$ ) über dieses Thema.)

Der Färber hat durch die Ammoniak-Hydrosulfitküpe daher die Garantie, die Ware in tadellosem Zustande zu erhalten. Die zahlreichen Proben solcher Küpenfärbungen, die wir mikroskopisch untersuchen konnten, ließen keine Formveränderungen der Wollfaser erkennen.

Chemisch läßt sich die Einwirkung von Alkalien auf Wolle durch das erhöhte Anfärbevermögen gegenüber sauren Farb stoffen zum Ausdruck bringen. Bei der Ammoniakbehandlung bemerken wir nur eine geringe Zunahme der Anfärbbarkeit.

Für die Wollfärberei ergibt sich aus ctem oben Gesagten die wichtige Folgerung, daß bei ,,doppelten " Färbungen, d. h. wo

8) W i t t, Chem. Technol. d. Gespinstfasern S. 89-91.

9) Färber-Ztg. (Lehne) 26, 269-272 [1915]; Angew. Chem. 29, II, 51 [1916]. 
alkalische (Küpen) und saure Färbebäder (saure Färbung oder Beizenfärbung) nacheinander in Anwendung kommen, wie z. B. bei der dunklen Melierwolle vieler Feldgraumelangen, die allergrößte Sorgfalt beobachtet werden muß, wenn keine Schädigung der Ware eintreten soll. Daß bei Bะachtung aller Vorsichtsmaßregeln auch praktisch bewährte Tuche erhalten werden, ist durch die jahrzehntelange Anwendung solcher Färbeweisen für Militär- und Ziviltuche bewiesen.

Unsere Mikrophotographien geben, um die Wirkung der verschiedenen Medien besonders deutlich hervortreten $\mathrm{zu}$

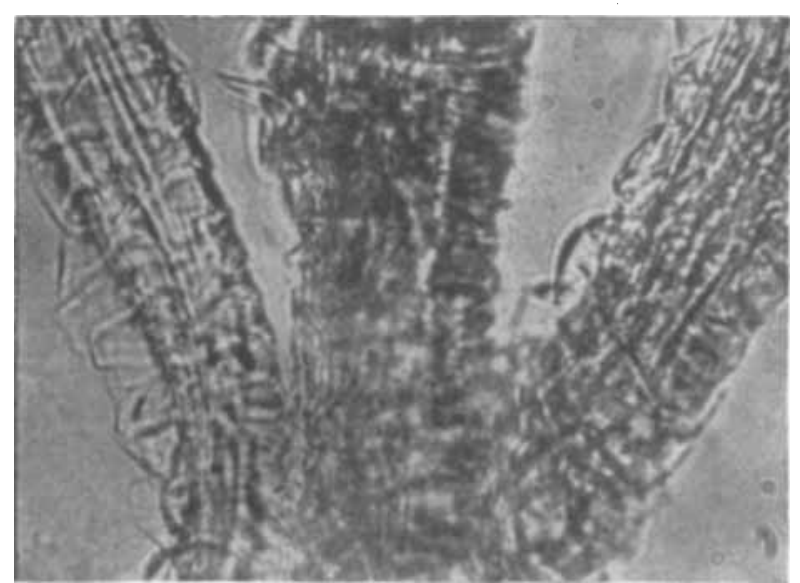

Bild 6. Wollfaser, $1 / 4$ Stunde mit $1 \%$ iger Natronlauge behandelt. Temperatur $80^{\circ}$.

lassen, daher nur Grenzzustände wieder, wie sie bei sachgemäßer Leitung eines Färbereibetriebes selbstredend nicht eintreten sollten; immerhin sind aber solche Schädigungen nicht ganz ausgeschlossen, wenn unvorsichtig gearbeitet, also $z$. B. die Küpe $\mathrm{zu}$ alkalisch und $\mathrm{zu}$ heiß geführt wird. Auch läßt die geringe Haltbarkeit mancher feldgrauen $\mathrm{Me}$ langen, auf die auch von anderer Seite des öfteren hingewiesen ist, wohl auf falsche oder unvorsichtige Behandlung der Ware schließen.

In dem eingangs zitierten Bericht versucht v. A l l w ö r d e $\mathrm{n}^{10}$ ), den Ursachen der Wollschädigungen durch zu

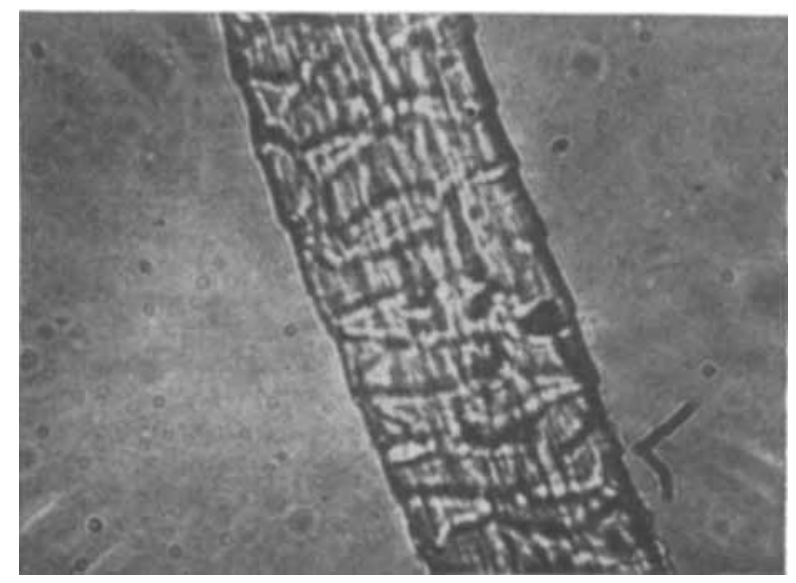

Bild 7. Wollfaser, $1 / 4$ stunde mlt $1 \%$ iger Natronlauge bei $40^{\circ}$ behandelt, dann 12/4 Stunde mit 1\%iger Schwefelsäure gekocht. (Beispiel fitr doppelte Färbung.)

stark alkalische Vorbehandlung und nachheriges Ausfärben in sauren Färbebädern eine interessante chemische Deutung zu geben.

Nach v. A ll w ö r d e n ist nur die äußere Epithelschicht der Wollfaser in gewissen Grenzen widerstandsfähig gegen Säuren, in Gegensatz zu den. Faserzellen der Rindenschicht. Außerdem befindet sich nach v. Allwörden zwischen Epithel- und Faserzellen ein kohlenhydratartiger Schutzkörper, das „Elasticum“, das bei alkalischen Wasch- und Färbeprozessen herausgelöst werden soll. Ist das Elasticum

10) Angew. Chem. 29, I, 77-78 [1916]. durch zu stark alkalische Behandlung der Wolle entzogen, so werden die empfindlichen, inneren Faserzellen der schädigenden Wirkung von Säuren preisgegeben.

v. Allw ö rde n weist das Elasticum auf mikrochemischem Wege durch die Chlorwasserreaktion nach, eine Reaktion, die in der Tat bei geschädigter Wollfaser ausbleibt.

v. A l I w ö r d e n kommt ebenfalls zu dem Schluß, daß die Zerreißfestigkeit eines Tuches allein noch keine Anhaltspunkte für seine Tragfähigkeit bietet. Durch stärkere Walke kann selbst bei weitgehender Schädigung der Wollfaser eine hohe Zugfestjgkeitsziffer erreicht werden, ohne daß die Tragechtheit genügt.

In solchen Fällen soll die Elasticumreaktion zur sicheren Erkennung fehlerhafter Ware dienen können.

Vermieden werden diese Gefahren durch vorsichtige, nicht zu alkalische Wäsche und eine e i $\mathrm{n} f \mathrm{a} \mathrm{ch}$ e Färbemethode.

Auch unsere Bilder (Nr. 7-9) zeigen, daß in der Tat bei d o p pelten Färbungen bei unsachgemäßer Führung des Wasch- und Färbeprozesses derartige bedenkliche Schädigungen der Wollfaser eintreten können.

Andererseits muß man sich unseres Erachtens auch vor zu weitgehenden Schlüssen aus der Elasticumtheorie hüten, und es wäre durchaus falsch, jede alkalische Färbeweise auf Grund solcher Überlegungen ohne weiteres zu verwerfen.

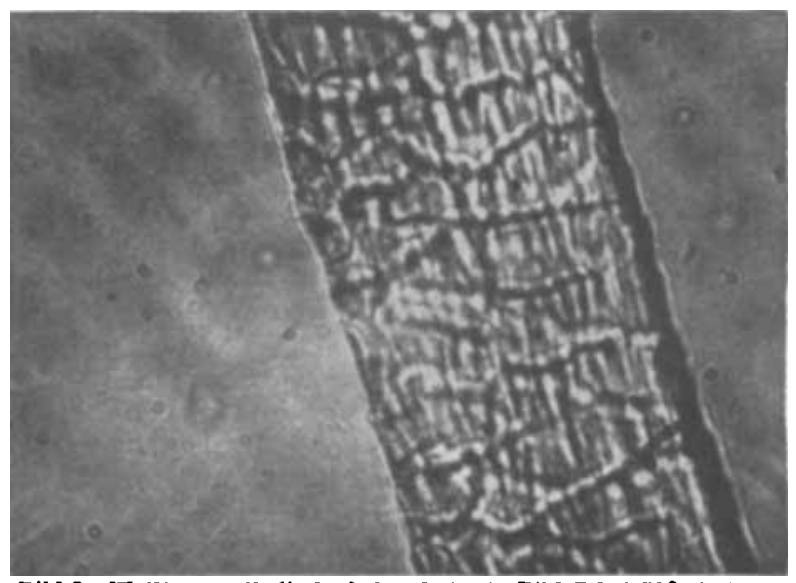

Blld 8. Wollfaser, alkalisch behandelt wie Blld 7 bei $60^{\circ}$, dann sauer behandelt whe Bild 7 .

Jedenfalls beweisen unsere Untersuchungen wiederum die schädliche Einwirkung zu starker alkalischer Behandlung auf die Wollfaser. Eine solche Behandlung braucht aber nicht allein die alkalische Küpen $f$ ä $r$ b e weise $z u$ sein, sondern jede zu stark alkalische Behandlung, der die Wolle unterworfen wird, kann Unheil anrichten. So können auch z: B. durch unsachgemäßes Waschen der Wolle oder unsachgemäßes Neutralisieren carbonisierter Wolle Schädigungen der Wollfaser eintreten, die, ähnlich wie bei den ,doppelten" Färbungen, durch das darauffolgende saure Ưberfärben noch vergrößert werden können.

Bei vorsichtig mit schwachen Sodalösungen gewaschenen Wollproben, wie wir sie z. B. aus württembergischen Tuchfabriken erhielten, konnten wir solche Schädigungen der Faser selbst bei starken Vergrößerungen mikroskopisch nicht feststellen.

Die Küpenfärbung gehört immer noch zu den ältesten und bewährten Färbeweisen und hat sich in der Militärbekleidung als Indigofärbung und als Küpenfeldgraufärbung durchaus gut bewährt. Treten aber einmal bei einer küpenfarbigen Ware Schädigungen der Wollfaser auf, so dürfte der Fehler nicht allein in der Färbeweise zu suchen sein, wie es heute fast immer geschieht, sondern auch die anderen $\mathrm{Be}$ handlungsweisen, bei. denen Alkali auf die Wollfaser einwirken kann, sind zu berücksichtigen.

So erfolgt das Waschen der Wolle im Inlande meist mit Soda, und es ist durchaus keine Seltenheit, daß mit bis zu 10\% igen Sodabädern gewaschen wird. Es leuchtet wohl ein, da $\beta$ bei solchen Konzentrationen Schädigungen der Woll- 
faser nicht nur möglich, sondern nach oben angeführten mikroskopischen Untersuchungen wahrscheinlich sind.

Dafür spricht auch eine Tatsache: englischen Stoffen wird heute noch nachgerühmt, daß sie besser in der Qualität sind als deutsche. Nun ist es bekannt, daß in England die Wollwäsche in der Regel nicht mit so viel Soda wie in Deutschland, sondern mit Soda und Seife erfolgt. Es ist nicht von der Hand zu weisen, daß vielleicht in der dadurch bedingten Schonung der Wollfaser die Utberlegenheit der englischen Stoffe beruht. In der Färbeweise kann dieser Unterschied nicht begründet sein, sind doch die englischen Fabrikanten auf die deutschen Farbstoffe und damit auf die gleichen Färbeweisen angewiesen, wie sie von deutschen Fabrikanten benutzt werden.

Vielleicht geben die vorliegenden Untersuchungen und Uberlegungen hier und dort eine Anregung zu Versuchen, um „englische" Qualitätsstoffe auch in Deutschland herzustellen.

\section{B a u m olle.}

a) Verhalten gegen $S$ äuren.

Utber das Verhalten der Baumwolle gegen Säuren liegt eine große Anzahl von Veröffentlichungen vor, die ausführlich ${ }_{\text {im }}$ im eingangs erwähnten Handbuch von S $\mathrm{ch}$ wa l be besprochen werden.

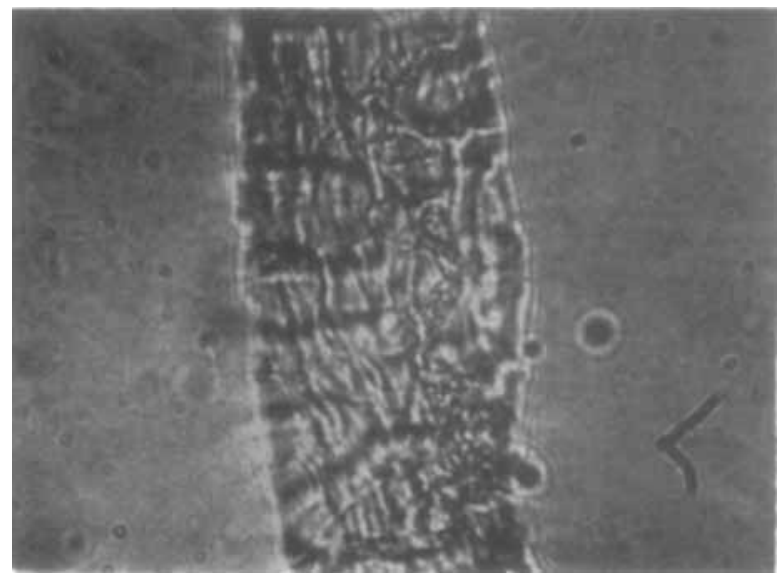

Bild 9. Wollfaser, alkalisch behandelt wie Bild 7 bel $60^{\circ}$, dann sauer behandelt wie Bild 7

Säuren bewirken die Bildung der leichtzerreiblichen Hydrocellulose. Letztere ist somit die Ursache des Mürbewerdens der Baumwollfaser bei der Behandlung mit Säuren. Im Gegensatz zur Wolle wird Baumwolle bereits von verdünnten Säuren stark angegriffen.

Mikroskopisch konnten wir eine Strukturveränderung der Baumwollfaser mit Säuren nicht feststellen. Die Baum. wollfaser ist eben ein einzelliges Gebilde und besteht nicht aus verschiedenartigen Zellen wie die Wolle, bei der wir die Auflösung in die verschiedenen Gewebsteile deutlich verfolgen können.

Auch nach $Z$ änker und $S c h n a b e 1^{11}$ zeigt das Mikroskop bei Baumwolle, die mit schwacher Schwefelsäure behandelt wurde, keine deutlichen Veränderungen oder Quellungen an.

b) Verhaltengegen Alkalien.

Gegen Alkalien ist die Baumwolle äußerst beständig. Erst bei höherer Konzentration findet eine Einwirkung statt, von der man beim sog. Mercerisieren praktisch Gebrauch macht.

Kochende, verdünnte Alkalien lösen jedoch bei Luftzutritt Baumwolle schließlich vollständig unter Bildung von Oxycellulose auf. Schließt man die Luft bei der Behandlung von Baumwolle mit Alkalien aus, so bleibt sie unverändert,

11) Dr. W. Z a n ker und K. S c h n a bol, Uber den Nachweis von freier Schwefelsäure auf Baumwolle. Färber-Ztg. (Lehne) 24, 260-263, 280-282 [1913]; Angew. Chem. 27, II, 31 [1914]. wenigstens zeigt uns das Mikroskop keine Formveränderungen an.

Chemisch läßt sich die Oxycellulose jedoch leicht nachweisen durch die stärkere Anfärbbarkeit mit Methylenblau.

\section{L e in en.}

Die Leinenfaser unterscheidet sich chemisch nur wenig von der Baumwollfaser. Sie ist gegen Schwefelsäure widerstandsfähiger als diese, aber weit weniger gegen Alkalien ${ }^{12}$ ).

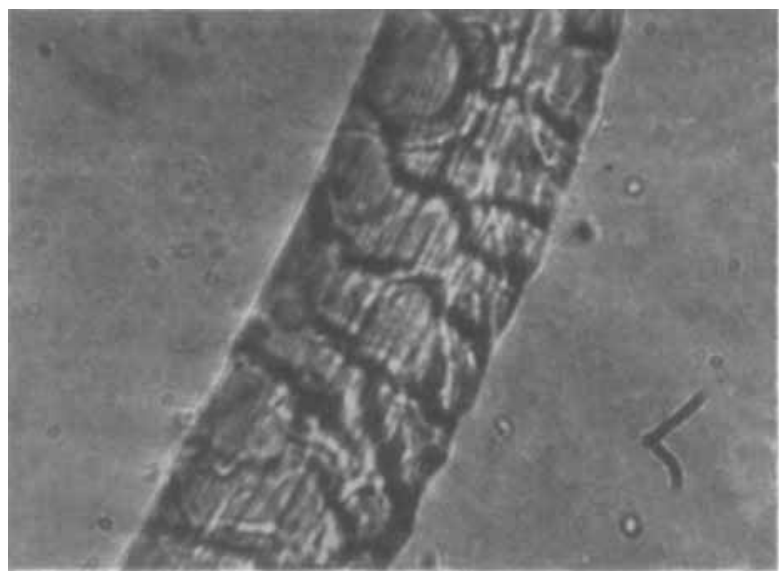

Bild 10. Wollfuser, mit $1 \%$ iger Schwefelgäure $11 / 4$ Stunde gekocht. (Beippiel für einfache saure Farbung.)

Wir haben ungebleichte und gebleichto Leinenfaser mit Säuren und Alkalien unter den gleichen Bedingungen behandelt, wie die vorhergehenden Fasern, und mikroskopisch untersucht. Jedoch versagt auch hier das Mikroskop, denn die Veränderungen sind wie bei der Baumwolle rein chemischer Natur und nicht von Strukturänderungen begleitet.

Fassen wir die Ergebnisse der Untersuchungen żusammen, so können wir auf die große Bedeutung des Mikroskops für die Beurteilung des Faserzustandes hinzweisen. Ganz besonders wertvoll wird die mikroskopische Untersuchung

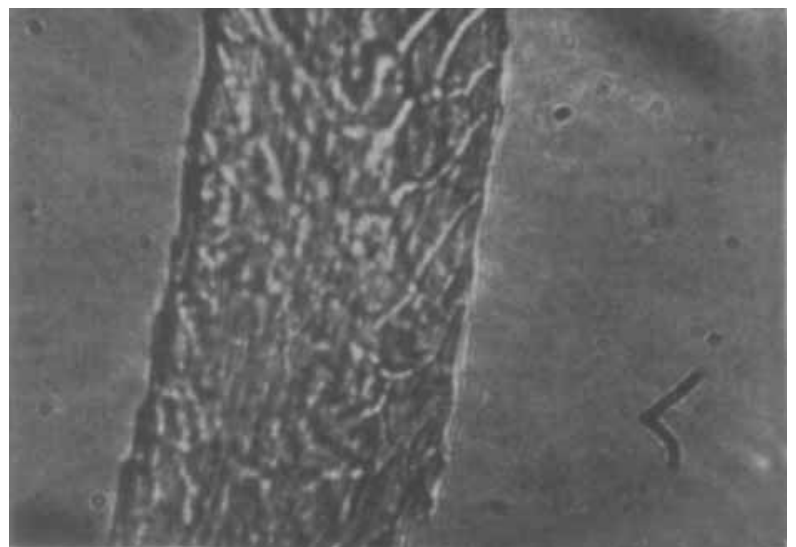

Bild 11. Wollfaser, nach Vorschrift mit Schwefelsăure gekocht und nachchromiert. (Beispiel fur Chromierfärbung.)

bei starken Vergrößerungen für die Verarbeitung der Wolle.

Die mechanische Prüfung der Textilwaren läßt nicht in allen Fällen ein einwandfreies Urteil über die jewejlige Trag. echtheit zu. Hier kann nun die mikroskopische und mikrochemische Untersuchung (wie die v. A ll w örd e n sche) wertvolle Aufschlüsse geben.

Außerdem ist $z u$ erwarten und $z u$ wünschen, daß auch die mechanischen Prüfungsmethoden immer mehr vervollkommnet werden, so daß die Textilindustrie über die Mittel verfügt, die Güte und Echtheit einer Ware jederzeit einwandsfrei zu beurteilen.

[A. 69.]

12) Schwalbe, Chemie der Cellulose. S. 581. (Berlin 1911.) 